\title{
Avaliação pericial da embriaguez: legislação e aspectos práticos
}

\author{
Forensic evaluation of alcoholic intoxication: legislation and practical \\ aspects
}

\author{
Luiz Lippi Rachkorsky¹, Talita Zerbini², Raquel Barbosa Cintra²
}

\begin{abstract}
Rackkorsky LL, Zerbini T, Cintra RB. Avaliação pericial da embriaguez: legislação e aspectos práticos. Saúde, Ética \& Justiça. 2012;17(2):44-9.

RESUMO: Após acidente de trânsito de carro versus moto, sem vítima fatal, o condutor do primeiro veículo foi encaminhado para exame de verificação de embriaguez, pois testemunhas referiram que o mesmo havia cruzado o sinal vermelho. Trata-se de homem de 37 anos, previamente hígido, que referia ter ingerido três latas de cerveja e duas doses de conhaque duas horas antes do exame. Ao exame físico pericial, constatou-se ataxia índex-index, hálito etílico e congestão cefálica, sem outras alterações da esfera psíquica e neurológica. Após o consentimento do periciando, foi realizada a coleta de amostra de sangue, que resultou em 2,8 gramas de álcool por litro de sangue $(2,8 \mathrm{~g} / \mathrm{L})$. De acordo com a literatura médico-legal, indivíduos com tal valor de alcoolemia geralmente apresentam inabilidade para ficar em pé e andar, desorientação, confusão e torpor. Desse modo, pergunta-se: é possível estabelecer uma correlação segura entre as alterações encontradas ao exame físico de verificação de embriaguez e o valor real de alcoolemia do indivíduo examinado? O presente relato exemplifica a dificuldade que a Lei 11.705/08 (Lei Seca) impôs ao médico-legista, pois muitas vezes o perito é questionado pelas autoridades judiciais e policiais se o examinado apresentava alcoolemia maior ou igual a $0,6 \mathrm{~g} / \mathrm{L}$ no momento dos fatos para permitir o correto enquadramento legal. De acordo com a legislação vigente, apenas os resultados do etilômetro e da alcoolemia são aceitos para fins de julgamento criminal. Porém, está sendo julgada no Supremo Tribunal Federal a legalidade de outros meios de provas para atestar embriaguez, sendo o exame clínico pericial a ênfase da discussão, uma vez que a prova produzida seria idônea para obter indícios de materialidade para instaurar a ação penal. Os autores discutem, baseados em literatura científica, os elementos médico-legais que podem ser utilizados no exame clínico e as demais formas de constatação de embriaguez por ingestão aguda de álcool etílico.
\end{abstract}

DESCRITORES: Intoxicação alcoólica; Transtornos do sistema nervoso induzidos por álcool; Consumo de bebidas alcoólicas/sangue; Prova pericial/utilização; Medicina legal; Literatura de revisão como assunto.

\footnotetext{
1 Médico Residente de Medicina do Trabalho da Faculdade de Medicina da Universidade de São Paulo.

2 Médica preceptora da Residência de Medicina do Trabalho e de Medicina Legal da Faculdade de Medicina da Universidade de São Paulo.

Endereço para correspondência: Luiz Lippi Rachkorsky. Avenida Doutor Arnaldo, 455, Cerqueira César-SãoPaulo, SP. CEP 012903-000. Email: luizIr@gmail.com
} 


\section{INTRODUÇÃO}

$\mathrm{O}$ $s$ acidentes de trânsito relacionados ao uso de álcool e outras drogas são considerados um problema de saúde pública mundial, devido ao grande número de vítimas ${ }^{1}$. A Organização Mundial da Saúde, em 2004, através da Aliança Global para a Segurança no Trânsito, apontou medidas que são capazes de diminuir a mortalidade nos acidentes, como obrigatoriedade do uso de equipamentos de segurança, cuidados no transporte de crianças, observação aos limites de velocidade e imposição de limites ao consumo de bebidas alcóolicas pelos motoristas ${ }^{2}$.

Em junho de 2008, seguindo uma tendência mundial, o Brasil buscou uma maneira mais rígida e direta de combater o uso de bebidas alcoólicas e direção, alterando o artigo 306 do Código de Trânsito Brasileiro (CTB $)^{3}$, que prevê crime caso o condutor esteja dirigindo com concentração igual ou superior a seis decigramas de álcool por litro de sangue. Esta mesma lei também alterou o artigo 277 do CTB $^{4}$, que vigora da seguinte maneira:

\begin{abstract}
"o condutor de veículo automotor, envolvido em acidente de trânsito ou que for alvo de fiscalização de trânsito, sob suspeita de dirigir sob a influência de álcool será submetido a testes de alcoolemia, exames clínicos, perícia ou outro exame que, por meios técnicos ou científicos, em aparelhos homologados pelo CONTRAN, permitam certificar seu estado's,4.
\end{abstract}

Desse modo, as pessoas são abordadas por policiais em blitz, sendo averiguado se o motorista está dirigindo embriagado por meio de uma breve entrevista, momento em que é verificado se existem os notórios sinais de embriaguez, que, de acordo com a Associação Brasileira de Medicina de Tráfego e o Conselho Nacional de Trânsito 5 , são: sonolência, hálito com odor alcoólico, olhos vermelhos, agressividade, dispersividade, exaltação, fala alterada, dificuldade de equilíbrio, se sabe onde está, entre outros. Ao identificar que o motorista apresenta algum dos sinais, o policial solicita que faça o teste do etilômetro. Se houver recusa, há a possibilidade de que ele seja conduzido ao Instituto Médico Legal ou repartição equivalente, após requisição de exame pelo Delegado de Polícia, a fim de colher sangue para exames laboratoriais ou ser submetido a exame clínico pelo perito médico-legal.

Entretanto, criou-se a equivocada interpretação de que, se o motorista se negar a realizar os exames, estará impune de sanções penais, fundamentada no principio constitucional e tratado internacional de que "ninguém é obrigado a produzir prova contra si mesmo"6, como seria o caso da prova que seria produzida pelo etilômetro ou pelo exame de sangue. Esta discussão ainda está sem desfecho, entretanto muitos juristas têm o mesmo pensamento e interpretação: "O motorista surpreendido, como se vê, pode recusar duas coisas: exame de sangue e bafômetro. Não pode recusar o exame clínico"7.

Desse modo, visto que o condutor não pode ser obrigado a fornecer amostra de sangue, a conclusão pericial deve ser baseada no exame clínico realizado pelo médico legista. Porém, em muitas ocasiões as autoridades judiciais ou policiais solicitam ao médico legista que realize equivalência entre as alterações do exame clínico e o nível de alcoolemia esperado para o momento dos fatos. Desse modo, o presente trabalho pretende discutir se há correlação segura entre os níveis de alcoolemia e as alterações de exame clínico que podem ser encontradas durante o exame médico pericial.

\section{OBJETIVOS}

Os autores pretendem responder à seguinte questão: é possível estabelecer uma correlação segura entre as alterações encontradas ao exame físico de verificação de embriaguez e o valor real de alcoolemia do indivíduo examinado?

\section{MÉTODOS}

Foi realizada revisão bibliográfica em publicações de 1985 a 2012 com os descritores "intoxicação alcoólica", "transtornos do sistema nervoso induzidos por álcool" e "medicina legal", além de pesquisa em jurisprudências disponíveis online. Além disso, será exposto um caso de avaliação de embriaguez que motivou os autores a realizar a referida revisão.

\section{RELATO DO CASO}

Após acidente de trânsito de carro $x$ motocicleta, sem vítima fatal, o condutor do veículo foi abordado por policiais e recusou-se a fazer o exame do etilômetro, sendo encaminhado para exame de verificação de embriaguez.

Chegando ao departamento médico-legal, o perito realizou o exame pericial no condutor: homem, 37 anos, previamente hígido, que referia ter ingerido três latas de cerveja e duas doses de 
conhaque duas horas antes do exame. Negou o uso de drogas e de medicação. Ao exame físico pericial constatou-se apenas ataxia índex-índex, hálito etílico e congestão cefálica, sem outras alterações da esfera psíquica e neurológica. A princípio o examinado se negou a colher sangue para exame, porém, após alguns instantes, permitiu a coleta do sangue para quantificação da concentração de álcool no sangue, que resultou em alcoolemia de 2,8 gramas de álcool por litro de sangue.

\section{DISCUSSÃO}

De acordo com o Decreto $n^{\circ} 6.488$ de 2008 , que regulamenta o artigo 276 e 306 da Lei $n^{\circ} 9.503$ de $1997^{\circ}$, o condutor que estiver sob a influência de álcool sofre sanções dependendo do valor da alcoolemia:

- alcoolemia menor que 0,2g de álcool por litro de sangue: sem sanções;

- alcoolemia maior que $0,2 \mathrm{~g}$ de álcool por litro de sangue e menor que 0,6 g de álcool por litro de sangue: sanções administrativas;

- alcoolemia maior que $0,6 \mathrm{~g}$ de álcool por litro de sangue: sanções administrativas e penais.

Em outras palavras, para que o infrator seja punido corretamente, é necessário saber qual sua alcoolemia no momento do fato. Entretanto, como ele pode se recusar a fazer o teste do etilômetro e a fornecer sangue para análise, o médico perito se encontra em uma situação complexa, sendo solicitado pelas autoridades judiciais e policiais a estimar a alcoolemia com base somente no exame clínico.

Antes de fazermos críticas à legislação, é necessário apontarmos que, segundo Andreuccetti et al. ${ }^{9}$, após a análise de um total de mais de 1.500.000 acidentes de trânsito no Estado e na cidade de São Paulo, evidenciou-se que, com a mudança da lei, houve uma redução significativa nas taxas de lesões e mortes em decorrência destes acidentes. Outro estudo comparativo realizado na cidade de Belo Horizonte, no estado de Minas Gerais, por Salgado², evidenciou uma redução de cerca de $50 \%$ na prevalência de condutores dirigindo com algum nível de álcool no sangue em 2008, quando comparados a 2007, o que coincide com a mudança na legislação e corrobora com a análise realizada em São Paulo. Ou seja, a mudança na lei mostrou-se uma importante ferramenta na prevenção de acidentes de trânsito em ambas as regiões.

Existe grande dificuldade em correlacionar sinais e sintomas de embriaguez com a alcoolemia $^{10,11}$. Nos artigos há a discussão de que a origem dos dados dos estudos que avaliaram essa capacidade, datados principalmente dos anos 80 e 90 , são obscuros, possuem referência pobre e que não consideraram fatores de confusão comuns, como a tolerância ao álcool ${ }^{10}$.

Esses estudos mostram diversas ferramentas e tabelas usadas para a constatação de sinais clínicos de embriaguez e correlação com concentração sanguínea, conforme mostra a Tabela 1.

TABELA 1 - Alcoolemia correspondente aos sinais encontrados em exame de verificação de embriaguez

\begin{tabular}{l|c|l}
\multicolumn{1}{c|}{ Sinal } & \multicolumn{1}{c}{ Alcoolemia(g/L) } & \multicolumn{1}{c}{ Principal Estudo } \\
\hline Olho vermelho & $>0,65$ & Mcknight et al. (2002) \\
\hline Odor de álcool & $>0,80$ & Moskowitz, Burns e Ferguson (1999) \\
\hline Distorções na fala & $>1,00$ & Klingholz, Penning and Liebhart (1988) \\
\hline Dificuldade para andar & $>2,00$ & Penttilla et al. (1974) \\
\hline Teste de Romberg positivo & $>1,50$ & Penttilla et al. (1974) \\
\hline Índex-nariz e índex-index & $>1,80$ & Widmark (1981) \\
\hline Hand Pat & $>1,20$ & Mcknight (1999) \\
Fonte: Rubenzer & &
\end{tabular}

Cherpitel et al. ${ }^{12}$ examinaram o diagnóstico de intoxicação alcoólica aguda em serviços de emergência e a relação com a concentração sanguínea de álcool. No estudo, foram examinadas 4.798 pessoas em 12 países. Era provido um rápido questionário, ao examinado, com uma lista de nove sintomas de intoxicação alcoólica aguda e para classificar entre intoxicação leve até intoxicação severa. Depois, os avaliadores aplicavam um questionário com questões padronizadas sobre 
o consumo e uso recente de álcool. No exame foi usado um check list que graduava entre não existente até muito severo os seguintes achados: odor de álcool, fala arrastada, diferença no volume da fala, diminuição da atenção, sudorese, respiração lenta, sonolência e olhos vermelhos. A autora indica que $84,6 \%$ dos entrevistados com alcoolemia de $0,6 \mathrm{~g} / \mathrm{L}$ de sangue ou mais foram classificados como intoxicados e 93,4\% abaixo desse nível foram classificados como não intoxicados. Oitenta por cento dos voluntários aparentemente não havia consumido bebida alcoólica, porém o artigo não é muito claro se esse número foi baseado em relato da própria pessoa ou em outra fonte de informação. A acurácia foi muito menor no grupo que reportou ter bebido em um intervalo de 6 horas antes do exame, tendo taxa de falha abaixo de $30 \%$ para alguns grupos, com média geral de $39 \%$. Em outras palavras, a alta acurácia aparentemente se deve à alta taxa de pessoas sóbrias ou que revelaram não ter consumido álcool. Outro ponto interessante no estudo é que ele relata que pessoas tolerantes ao álcool podem não ser detectadas.

Os sinais e sintomas da intoxicação alcoólica geralmente se manifestam seguindo os níveis sanguíneos, da seguinte maneira ${ }^{13}$.

TABELA 2 - Alcoolemia e efeitos no organismo

\begin{tabular}{c|l}
\hline $\begin{array}{c}\text { Alcoolemia } \\
(\mathbf{g} / \mathbf{L})\end{array}$ & \multicolumn{1}{c}{ Efeitos } \\
\hline $0,1-0,5$ & $\begin{array}{l}\text { Aumento do ritmo cardíaco e respiratório; diminuição da atenção; Comportamento incoerente ao } \\
\text { executar tarefas; diminuição da capacidade de julgamento e perda de inibição; leve sensação de } \\
\text { euforia, relaxamento e prazer. }\end{array}$ \\
\hline $0,6-1,0$ & $\begin{array}{l}\text { Diminuição da atenção, julgamento e controle; reflexos mais lentos; dificuldade de coordenação } \\
\text { e redução da força muscular; redução da capacidade de tomar decisões ou de discernimento; } \\
\text { sensação crescente de ansiedade e depressão. }\end{array}$ \\
\hline $1,0-1,5$ & $\begin{array}{l}\text { Reflexos consideravelmente mais lentos; problemas de equilíbrio e movimento alterados; alteração } \\
\text { de algumas funções visuais; dificuldade na fala; vômito, sobretudo se esta alcoolemia for atingida } \\
\text { rapidamente. }\end{array}$ \\
\hline $1,6-2,9$ & $\begin{array}{l}\text { Alterações graves da coordenação motora, com tendência a cambalear e a cair frequentemente; } \\
\text { estado emocional exagerado (medo, aborrecimentos, aflição); distúrbio da sensação e da percepção } \\
\text { às cores, formas, movimentos e dimensões; debilidade no equilíbrio; incoordenação muscular. }\end{array}$ \\
\hline $3,0-3,9$ & $\begin{array}{l}\text { Letargia profunda; perda de consciência; estado de sedação comparável ao de uma anestesia } \\
\text { cirúrgica. }\end{array}$ \\
\hline$>4,0$ & $\begin{array}{l}\text { Inconsciência; incontinência urinária e fecal; parada respiratória; morte, em geral provocada por } \\
\text { insuficiência respiratória. }\end{array}$ \\
\hline
\end{tabular}

Adaptado de: Dubowski ${ }^{14}$, Homburger ${ }^{15} ;$ Amdur $^{16}, \mathrm{WHO}^{17}$.

Segundo Salgado","o entrevistador não foi capaz de identificar que o entrevistado estava sob influência de álcool em quase $70 \%$ dos casos"2. Em seu estudo, os observadores não foram capazes de detectar índices de alcoolemia leve a moderada.

Campos $^{1}$ mostra que $19,6 \%$ dos entrevistados excediam os limites permitidos (maior que $0,6 \mathrm{~g} / \mathrm{L}$ ). Entretanto, ele aponta que apenas $20,5 \%$ desses foram identificados como estando sob efeito de bebidas alcoólicas. Ou seja, quase $80 \%$ das pessoas que haviam ingerido álcool não apresentavam sinais de embriaguez.

Os trabalhos analisados apontam para grande divergência entre as alterações de exame clínico detectadas durante a verificação de embriaguez e os valores de álcool etílico no sangue ou no ar alveolar, o que aponta para a grande dificuldade que o médico legista enfrenta no momento em que lhe é solicitado realizar a estimativa da alcoolemia baseada no exame clínico.

\section{CONCLUSÃO}

O examinado do presente trabalho apresentava sintomatologia muito mais branda que a esperada pela alcoolemia constatada, o que nos permite concluir que correlacionar alterações de exame clínico com o valor de alcoolemia é uma tarefa difícil, visto que as pessoas apresentam manifestações clínicas e repercussões funcionais muito diversas. 
Rackkorsky LL, Zerbini T, Cintra RB. Avaliação pericial da embriaguez: legislação e aspectos práticos.

Para que não ocorra estimativa errada da alcoolemia por meio dos achados do exame clínico, faz-se necessária uma mudança na legislação: estabelecendo tolerância zero para o álcool nos exames de sangue e/ou no etilômetro ou considerando apenas o estado clínico de embriaguez à perícia médica. Desse modo, evitamse conclusões errôneas em decorrência das discrepâncias visualizadas entre exame clínico e alcoolemia.

Rackkorsky LL, Zerbini T, Cintra RB. Forensic evaluation of alcoholic intoxication: legislation and practical aspects. Saúde, Ética \& Justiça. 2012;17(2):44-9.

\begin{abstract}
After a crash between a car and a motorcycle, without any fatal victims, the driver of the car was referred by the police for blood alcohol screening, as witnesses reported that he ran a red light. He was 37 years old at the time, reported no previous illness and that he had drunk three cans of beer and two shots of cognac two hours before being evaluated by the medical examiner. The physical exam revealed ataxia index-index, alcohol on breath and head congestion, but no altered neurological or psychological mental status. After consent, a blood sample was collected, which resulted in a blood alcohol content (BAC) of a $2.8 \mathrm{~g} / \mathrm{L}$. According to the scientific literature, anyone with such BAC would exhibit inability to stand and walk, disorientation, confusion and drowsiness. This case challenges the correlation between blood alcohol levels and the effectiveness of certain physical responses, and exemplifies how difficult it is for the appointed medical examiner to provide judicial and police authorities with a clinical diagnosis of a suspect BAC greater than or equal to $0.6 \mathrm{~g} / \mathrm{L}$, under the requirements of Law 11.705/08 ("Lei Seca"). According to current legislation, only the results of the BAC and the breath alcohol concentration are accepted for purposes of criminal prosecution. However, the legality of other means of evidence to attest drunkenness is on trial in the Supreme Court, being the expert clinical examination the emphasis of the discussion, since the evidence produced would meet for obtaining evidence of materiality to bring criminal action. The medical-legal elements that can be used in clinical examination and other forms of verification of acute intoxication by ingestion of ethyl alcohol are disussed based on the scientific literature.
\end{abstract}

KEYWORDS: Alcohol intoxication; Alcohol-induced disorders, nervous system; Alcohol drinking/blood; Expert testimony/utilization; Forensic medicine; Review literature as topic.

\title{
REFERÊNCIAS
}

1. Campos VR, Salgado R, Dualibi S, Laranjeira R. Prevalência do beber e dirigir em Belo Horizonte, Minas Gerais, Brasil. Cad Saude Publica. 2008;24(4):82934.

2. Salgado RS, Campos VR, Dualibi S, Laranjeira RR. O impacto da "lei seca" sobre o beber e dirigir em Belo Horizonte/MG. Cien Saude Colet. 2012;17(4):971-6.

3. Brasil. Lei no 9.503, de 23 de setembro de 1997. Código de Trânsito Brasileiro. Diário Oficial da União, 23 set. 1997.

4. Brasil. Lei $n^{\circ} \mathbf{1 1 . 7 0 5}$, de 26 de junho de 2008 . Altera a Lei no 9.503, de 23 de setembro de 1997, que 'institui o Código de Trânsito Brasileiro', e a Lei no 9.294, de 15 de julho de 1996, que dispõe sobre as restrições ao uso e à propaganda de produtos fumígeros, bebidas alcoólicas, medicamentos, terapias e defensivos agrícolas, nos termos do $\S 4^{\circ}$ do art. 220 da Constituição Federal, para inibir o consumo de bebida alcoólica por condutor de veículo automotor, e dá outras providências. Diário Oficial da União, 16 jun. 2008.

5. Miranda JJ. Recusa de bafômetro é presunção de embriaguez. Rev Curso Direito FEAD Minas Gerais, PHRONESIS. 2006.

6. Brasil. Decreto $n^{\circ} \mathbf{6 7 8}$, de 06 de novembro de 1992. Promulga a Convenção Americana sobre Direitos Humanos (Pacto de São José da Costa Rica), de 22 de novembro de 1969. Diário Oficial da União, 06 nov. 1992.

7. Buzaglo AS. Considerações sobre a lei seca - alcoolemia - substâncias psicoativas e autoincriminação. Carta Mensal - RJ. 2012;676:30-52.

8. Brasil. Decreto no 6.488 , de 19 de junho de 2008 . Regulamenta os arts. 276 e 306 da Lei no 9.503, de 23 de setembro de 1997 - Código de Trânsito Brasileiro, disciplinando a margem de tolerância de álcool no sangue e a equivalência entre os distintos testes de alcoolemia para efeitos de crime de trânsito. Diário Oficial da União, 19 jun. 2008.

9. Andreuccetti G, Carvalho HB, Cherpitel CJ, Ye Y, Ponce JC, Kahn T, Leyton V. Reducing the legal blood alcohol concentration limit for driving 
Rackkorsky LL, Zerbini T, Cintra RB. Avaliação pericial da embriaguez: legislação e aspectos práticos.

in developing countries: a time for change? Results and implications derived from a time-series analysis (200110) inducted in Brazil. Addiction. 2011;106(12):2124-31.

10. Roberts JR, Dollard D. Alcohol levels do not accurately predict physical or mental impairment in ethanoltolerant subjects: relevance to emergency medicine and dram shop laws. J Med Toxicol. 2010;6(4):43842.

11. Rubenzer S. Judging intoxication. Behav Sci Law. 2011;29(1):116-37.

12. Cherpitel C, Bond J, Ye Y, Room R, Poznyak V, Rehm J, Peden M. Clinical assesement compared with breathalyzer readings in the emergency room: Concordance of ICD-10 Y90 and Y91 codes. Emerg
Med J. 2005;22(10):689-95.

13. Roldán J, Frauca C, Dueñas A. Intoxicación por alcoholes. Anales Sis San Navarra. 2003;26(Suppl.1):129-39.

14. Dubowski KM. Manual for analysis of ethanol in biological liquids. Washington, D.C.: U.S. Department of Transportation; 1977.

15. Homburger F, Hayes JA, Pelikan EW. A guide to general toxicology. Basel: Karger; 1983.

16. Amdur MO, Doull J, Klaassen CD. Casarett and Doull's toxicology: the basic science of poisons. New York: Pergamon Press; 1991.

17. World Health Organization. Drinking and driving: an international good practice manual. Genebra; 2007. 